Case Report

\title{
Unusual Presentation of Tuberculous Thyroid Abscess in a Background of Hashimoto's Thyroiditis in a Chronic Hepatitis B Carrier
}

\author{
Sakthivel Chinnakkulam Kandhasamy,, Sunil Kumar, ${ }^{1}$ Anubhav Sangwan, ${ }^{1}$ \\ Neelam Sahani, ${ }^{2}$ Gopalakrishnan Gunasekaran, ${ }^{1}$ Sunil Kumar Meena, ${ }^{1}$ \\ and Swapnil Singh Kushwaha ${ }^{1}$ \\ ${ }^{1}$ Department of General Surgery, Vardhman Mahavir Medical College and Safdarjung Hospital, New Delhi, India \\ ${ }^{2}$ Department of Pathology, Vardhman Mahavir Medical College and Safdarjung Hospital, New Delhi, India
}

Correspondence should be addressed to Sakthivel Chinnakkulam Kandhasamy; medicosurgeon@gmail.com

Received 29 July 2016; Accepted 6 December 2016

Academic Editor: Nisar A. Chowdri

Copyright (C) 2016 Sakthivel Chinnakkulam Kandhasamy et al. This is an open access article distributed under the Creative Commons Attribution License, which permits unrestricted use, distribution, and reproduction in any medium, provided the original work is properly cited.

\begin{abstract}
Tuberculosis of thyroid gland is a very rare disease. It has variable presentations and may be sometimes associated with autoimmune thyroiditis. We report a case of 45-year-old male, with left sided painless neck swelling, with a purulent discharging sinus over it associated with night sweats and loss of appetite. Thyroid imaging disclosed heterogeneous enhancement of left lobe of thyroid gland with internal vascularity and coarse calcifications. Core needle biopsy revealed caseous necrosis and AFB positivity. Patient had thyroid peroxidase antibody and thyroglobulin antibody positivity and the rest of thyroid function tests were normal. Patient had positive Mantoux test, hepatitis B surface Ag, and low viral DNA. The patient was diagnosed as being a case of tuberculous abscess of thyroid gland and was put on antitubercular therapy for 2 months. Patient subsequently underwent left hemithyroidectomy when there was no response. Histopathological examination revealed tuberculosis of thyroid gland. A final diagnosis of tuberculous abscess of thyroid gland in a background of Hashimoto's thyroiditis in a chronic HBV carrier was made. Therefore, although rare tuberculosis of thyroid should be kept in mind as a differential diagnosis of thyroid swelling.
\end{abstract}

\section{Introduction}

Tuberculosis of thyroid gland is extremely uncommon even in countries where the prevalence of tuberculosis is high. The exact number of cases reported is difficult to estimate; barely 200 cases can be found in literature [1]. The mycobacterium tuberculosis reaches the gland through blood and lymphatics or direct extension from cervical lymph nodes. It has variable presentation, but most cases manifest as a nodule or caseous abscess. The diagnosis can be made from FNAC (fine needle aspiration cytology) and culture of the aspirated material or sometimes by histopathological examination of resected gland.

Hashimoto's thyroiditis also known as chronic autoimmune lymphocytic thyroiditis is the most common autoimmune thyroid disease. It is multifaceted disease exhibit- ing various clinical presentations. It presents with painless enlargement of the thyroid gland and is associated with detection of thyroid autoantibodies [2]. Literature shows that the disease is 15 times more common in women with the peak incidence varying between the ages of 30 and $50[2,3]$. Environmental triggers, drugs, chemicals, and infection contribute to autoimmune thyroid disease. Thyroid involvement may be regarded as the most frequent alteration in HCV (hepatitis C virus) positive patients and is more frequent than in HBV (hepatitis B virus) positive patients. Thyroid autoimmunity in viral infection may be due to a cytokine-induced disease in susceptible patients [4].

We here present a case of 45 -year-old male with tuberculous thyroid abscess in a background of Hashimoto's thyroiditis in a chronic hepatitis B carrier. 


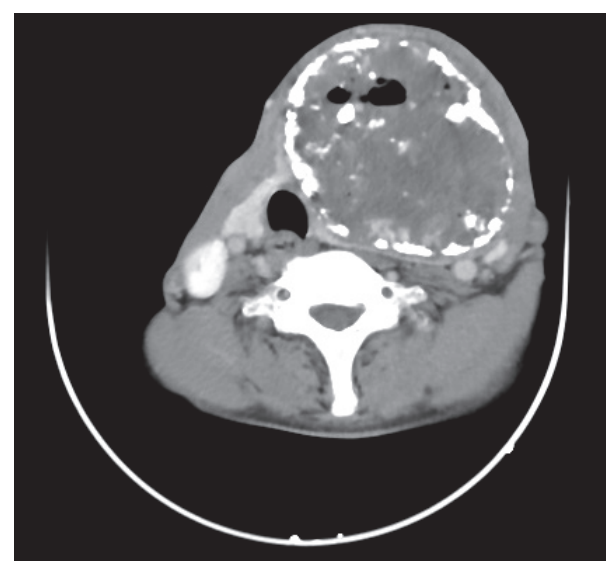

FIGURE 1: Computed tomography revealing enlarged left thyroid lobe with heterogeneous enhancement and calcifications. There was a hypodense component with focal air loculi within the lesion.

\section{Case Presentation}

A 45-years-old male, with no significant past medical history, presented to the outpatient department with 15-year history of painless neck swelling in the left side and 5-month history of purulent discharging sinus over it. The swelling was slowly progressive in nature and he had mild discomfort while swallowing. There was no complaint of dyspnea or hoarseness of voice. There was no specific history suggestive of hypoor hyperthyroidism. Though the patient denied any history of fever, he complained of night sweats and loss of appetite. Patient did not have any past history or family history of tuberculosis. Physical examination revealed enlarged left lobe of thyroid $(10 \times 8 \mathrm{~cm})$ with a single purulent discharging sinus over it with undermined edges. There were no palpable cervical lymph nodes. Pulmonary system examination was normal. Thyroid hormone assay revealed thyroid stimulating hormone (TSH) $2.99 \mu \mathrm{IU} / \mathrm{mL}$, thyroxine (T4) $9.08 \mu \mathrm{g} / \mathrm{dL}$, triiodothyronine (T3) $138 \mathrm{ng} / \mathrm{dL}$, thyroglobulin (Tg) $42 \mathrm{ng} / \mathrm{mL}$, thyroglobulin antibody (TgAb) $150 \mathrm{IU} / \mathrm{mL}$, and thyroid peroxidase antibody (TPOAb) $232 \mathrm{IU} / \mathrm{mL}$. Hematological examination revealed hemoglobin $12 \mathrm{~g} \%$, hematocrit $42 \%$, ESR $75 \mathrm{~mm} /$ first hour, platelets $196000 / \mathrm{mm}^{3}$, and WBC $9300 / \mathrm{mm}^{3}$, with normal differential count. Chest X-ray was normal but Mantoux test was positive $(20 \times 18 \mathrm{~mm})$. Viral markers test for HIV and anti-HCV was negative. But patient was positive for $\mathrm{HbsAg}$ with $\mathrm{HbeAg}$ negative and hepatitis B DNA titre of $799 \mathrm{IU} / \mathrm{mL}$. Thyroid ultrasonography revealed enlarged left lobe of thyroid with heterogeneous contents with calcifications. Contrast enhanced computed tomography was performed which showed heterogeneous enhancement of the left lobe of thyroid gland $(79 \times 95 \times 88 \mathrm{~mm})$ with internal vascularity and coarse calcifications. There was a hypodense component with focal air loculi within the lesion (Figure 1). FNAC showed focal necrosis against hemorrhagic background without demonstrable acid fast bacillus (AFB). Culture of the aspirated fluid for mycobacterium tuberculosis came out negative. Core needle biopsy was performed which

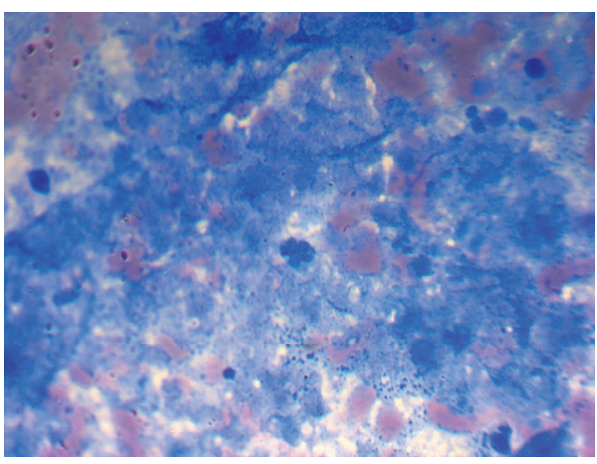

FIGURE 2: Core needle biopsy showing caseous necrosis and stain for AFB positive.

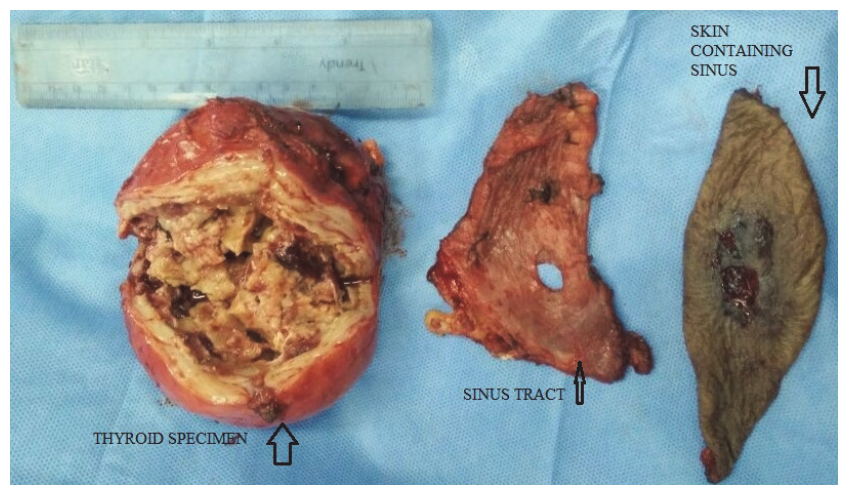

FIGURE 3: Cut section of thyroid showing greyish white color with extensive areas of autolysis and a cavity identified in the center of the lesion.

showed severe chronic inflammation, caseous necrosis, and stain for AFB positive (Figure 2). The patient was put on WHO category 1 antitubercular regime, that is, combination of rifampicin $450 \mathrm{mg}$, isoniazid $300 \mathrm{mg}$, ethambutol $1200 \mathrm{mg}$, and pyrazinamide $1500 \mathrm{mg}$ thrice a week for 2 months. After 2 months, patient had developed progression of swelling with pressure symptoms for which he underwent left hemithyroidectomy.

Grossly, the left thyroid lobe was enlarged measuring $8 \times$ $9 \times 8 \mathrm{~cm}$. The cut section was greyish white with extensive areas of autolysis and a cavity identified in the center of the lesion (Figure 3). On histopathological examination, there was destruction of thyroid follicles and extensive fibrosis at the periphery. Numerous lymphoid follicles were seen with lymphocytes infiltrating into the surrounding fibrocollagenous tissue. A $1 \mathrm{~cm}$ sinus tract was identified which was lined by dense inflammatory granulation tissue and fibrosis, features suggestive of Hashimoto's thyroiditis (Figure 4) and areas of extensive necrosis adjacent to thyroid follicles suggestive of tuberculosis of thyroid (Figure 5). Final diagnosis of tuberculous thyroid abscess in a background of Hashimoto's thyroiditis in a chronic hepatitis B carrier was made. Postoperatively patient was continued on antituberculous regimen for 9 months. 


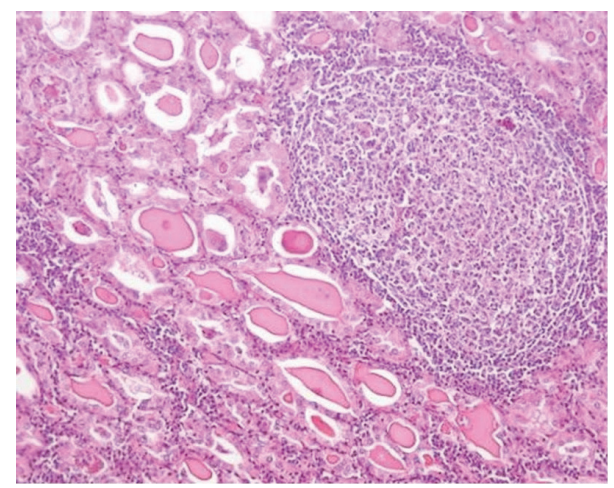

FIGURE 4: Thyroid histopathology showing destruction of thyroid follicles. Numerous lymphoid follicles were seen with lymphocytes infiltrating into the surrounding fibrocollagenous tissue.

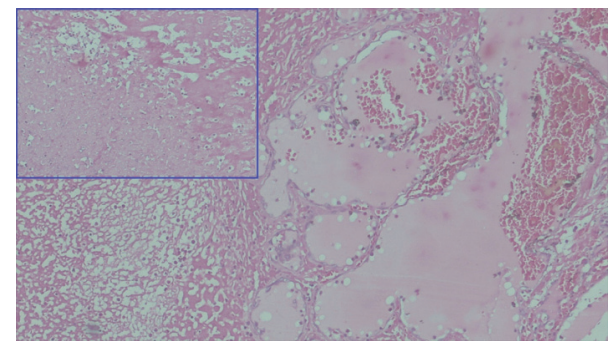

FIGURE 5: Tissue section showing areas of necrosis and adjacent thyroid follicles suggestive of tuberculosis of thyroid gland. Boxed area showing extensive areas of necrosis.

\section{Discussion}

Thyroid tuberculosis is an extremely rare, whether primary or secondary. Certain tissue in our body is resistant to tuberculosis including thyroid, cardiac muscle, skeletal muscle, and pancreas [5]. Thyroid gland is known to resist the infection but exact reason remains unclear. It is assumed that various reasons included the presence of bactericidal action of colloid material, thyroid capsule, antitubercular action of thyroid hormones, increased blood flow, and increased iodine which cause destruction of tubercle bacilli [6].

The incidence of thyroid tuberculosis is $0.1 \%$ to $0.6 \%$ in resected specimens [7]. Clinical presentation is variable and symptoms are often nonspecific. The patient may present with goitre and associated pressure symptoms like dyspnea, dysphagia, and hoarseness of voice. There may be a painless discharging sinus. Diagnosis can be made from FNAC with staining for AFB or culture of the aspirated material. The characteristic histological features include caseous necrosis, necrotizing epithelioid granuloma, and Langhans type giant cells. Demonstration of acid fast bacilli by Ziehl-Neelsen staining confirms the diagnosis but it is often negative in tissue section [8]. Thyroid tuberculosis must be differentiated from other granulomatous disorders. Sometimes patient may present with subacute granulomatous (De Quervain's) thyroiditis or chronic thyroiditis [9]. Our patient 45-year-old male who was a chronic hepatitis B carrier presented with tuberculous thyroid abscess in a background of Hashimoto's thyroiditis.
Previously, treatment of thyroid tuberculosis consisted of antituberculous drug and surgical removal of the gland [10]. Lately it has been found that antituberculous drug alone is sufficient to control the disease [11]. If the patient presents with large abscess, surgical drainage or resection is often necessary [12].

Hashimoto's thyroiditis is one of the most common thyroid disorders. The disease has been called Hashimoto's thyroiditis, chronic thyroiditis, lymphadenoid goitre, and recently autoimmune thyroiditis. In this disease there will be autoimmune destruction of thyroid glands associated with chronic lymphocyte infiltration, eosinophilic change, fibrosis, and atrophy of the thyroid gland. Patient may present with painless diffuse enlargement of thyroid gland. At times, there will be no goitre at presentation. It may be associated with hypothyroidism $[2,3]$.

In autoimmune thyroiditis, there is presence of thyroid autoantibodies in patients' sera against two major thyroid antigens (thyroid peroxidase and thyroglobulin). Thyroid peroxidase (TPO) is essential for various steps in thyroid hormone synthesis, iodine oxidation, iodination of tyrosine residues, and coupling of iodotyrosine. TPO is located at the apical membrane of the thyroid follicles. Thyroglobulin ( $\mathrm{Tg}$ ) is located within the follicles and helps in storage of thyroid hormones [13]. Antibodies against TPO (TPOAb) and $\mathrm{Tg}(\mathrm{TgAb})$ are able to cause destruction of thyroid cells due to antibody dependent cell cytotoxicity. These antibodies are useful in the diagnosis of thyroid autoimmunity. In Hashimoto's thyroiditis, TPOAbs are present in nearly all (>90\%) patients, while TgAbs can be found in $80 \%[14,15]$.

Viral infections play a role in the development of autoimmunity possibly by the molecular mimicry between viral and self-antigens. Viral infection can cause the release of proinflammatory mediators which may lead to activation of autoreactive T-cells [13]. Autoimmune phenomenon is more common with hepatitis $\mathrm{C}$ virus (HCV) than hepatitis $B$ virus (HBV). Therapy with interferon has important effects on expression of major histocompatibility antigens [16] and the regulation of cytokine production [17]. Autoimmunity against the thyroid gland in patients with hepatitis B infection is not well understood. Positive levels of TPOAb and TgAb were found in $20 \%$ and $11 \%$ of patients with HCV compared with $5 \%$ and $3 \%$ of patients with HBV, respectively [4]. Variable geographic distribution has also shown that genetic or environmental influences could be implicated [18]. On the whole, distinctive role of the virus itself or antiviral treatment remains to be clarified.

Hashimoto's thyroiditis patients need no treatment, because the disease is mostly asymptomatic, and often have small goitre [19]. Surgery is reserved for the patients who have significant pain, cosmetic, or pressure effects, after a trial of steroid therapy. Steroid therapy may result in resolution in some cases. If the patients have hypothyroidism, it needs thyroxine treatment [20].

\section{Conclusion}

Though a rare entity, thyroid tuberculosis should be considered in differential diagnosis of thyroid swelling presenting 
as abscess. FNAC and culture are the main diagnostic investigation. Sometimes, it may be associated with autoimmune thyroiditis. Treatment is mainly based on antituberculous drugs, but surgery is rarely required especially in cases presenting with large thyroid abscess.

\section{Competing Interests}

The authors declare that they have no conflict of interests.

\section{Acknowledgments}

The authors are grateful to the Medical Superintendent, Vardhman Mahavir Medical College, and Safdarjung Hospital, New Delhi, for allowing them to publish this case.

\section{References}

[1] I. Zendah, H. Daghfous, S. B. Mrad, and F. Tritar, "Primary tuberculosis of the thyroid gland," Hormones, vol. 7, no. 4, pp. 330-333, 2008.

[2] E. N. Pearce, A. P. Farwell, and L. E. Braverman, "Thyroiditis," New England Journal of Medicine, vol. 348, no. 26, pp. 2646$2655,2003$.

[3] I. D. Hay, "Thyroiditis: a clinical update," Mayo Clinic Proceedings, vol. 60, no. 12, pp. 836-843, 1985.

[4] R. Desailloud and D. Hober, "Viruses and thyroiditis: an update," Virology Journal, vol. 6, article no. 5, 2009.

[5] S. P. Kataria, P. Tanwar, S. Singh, and S. Kumar, "Primary tuberculosis of the thyroid gland: a case report," Asian Pacific Journal of Tropical Biomedicine, vol. 2, no. 10, pp. 839-840, 2012.

[6] F. Safarpor, M. H. Hedayati-Omami, F. Mohammadi, S. Hoda, and D. Safarpor, "Thyroid tuberculosis," Iranian Red Crescent Medical Journal, vol. 9, pp. 161-163, 2007.

[7] H. Kabiri, F. Atoini, and A. Zidane, "Thyroid tuberculosis," Annales d'Endocrinologie, vol. 68, no. 2-3, pp. 196-198, 2007.

[8] F. Abdulsalam, S. Abdulaziz, and A. A. Mallik, "Primary tuberculosis of thyroid gland," Kuwait Medical Journal, vol. 37, pp. 116-118, 2005.

[9] A. Ghosh, S. Saha, B. Bhattacharya, and S. Chattopadhay, "Primary tuberculosis of thyroid gland: a rare case report," American Journal of Otolaryngology-Head and Neck Medicine and Surgery, vol. 28, no. 4, pp. 267-270, 2007.

[10] V. K. Talwar, H. Gupta, and A. Kumar, "Isolated tuberculous thyroiditis," Journal, Indian Academy of Clinical Medicine, vol. 4, no. 3, pp. 238-239, 2003.

[11] F. Orlandi, S. Fiorini, I. Gonzatto et al., "Tubercular involvement of the thyroid gland: a report of two cases," Hormone Research, vol. 52, no. 6, pp. 291-294, 1999.

[12] U. Majid and N. Islam, "Thyroid tuberculosis: a case series and a review of the literature," Journal of Thyroid Research, vol. 2011, Article ID 359864, 4 pages, 2011.

[13] K. Zaletel and S. Gaberšček, "Hashimotos thyroiditis: from genes to the disease," Current Genomics, vol. 12, no. 8, pp. 576588, 2011.

[14] K. Zaletel, "Determinants of thyroid autoantibody production in Hashimoto's thyroiditis," Expert Review of Clinical Immunology, vol. 3, no. 2, pp. 217-223, 2007.
[15] S. M. McLachlan and B. Rapoport, "Why measure thyroglobulin autoantibodies rather than thyroid peroxidase autoantibodies?" Thyroid, vol. 14, no. 7, pp. 510-520, 2004.

[16] M. Saji, J. Moriarity, T. Ban, D. S. Singer, and L. D. Kohn, "Major histocompatibility complex class I gene expression in rat thyroid cells is regulated by hormones, methimazole, and iodine as well as interferon," The Journal of Clinical Endocrinology \& Metabolism, vol. 75, pp. 871-878, 1992.

[17] L. K. Chen, D. Matheiu-Mahul, F. H. Bach, J. Dausset, A. Bensussan, and M. Sasportes, "Recombinant interferon alpha can induce rearrangement of T-cell antigen receptor alphachain genes and maturation to cytotoxicity in T-lymphocyte clones in vitro," Proceedings of the National Academy of Sciences of the United States of America, vol. 83, no. 13, pp. 4887-4889, 1986.

[18] N. R. Rose, R. Bonita, and C. L. Burek, "Iodine: an environmental trigger of thyroiditis," Autoimmunity Reviews, vol. 1, no. 1-2, pp. 97-103, 2002.

[19] A. L. Vickery and E. Hamlin Jr., "Struma lymphomatosa (Hashimoto's thyroiditis)-observations on repeated biopsies in sixteen patients," The New England Journal of Medicine, vol. 264, pp. 226-229, 1961.

[20] B. Karges, R. Muche, I. Knerr et al., "Levothyroxine in euthyroid autoimmune thyroiditis and type 1 diabetes: a randomized, controlled trial," The Journal of Clinical Endocrinology \& Metabolism, vol. 92, no. 5, pp. 1647-1652, 2007. 


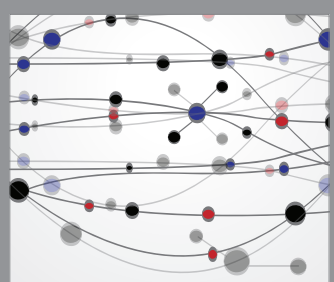

The Scientific World Journal
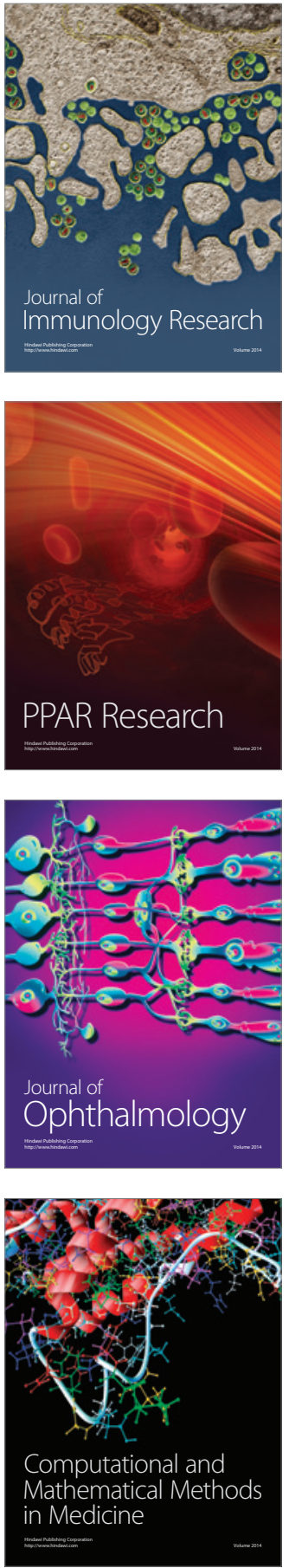

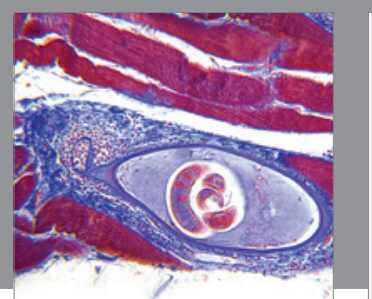

Gastroenterology Research and Practice

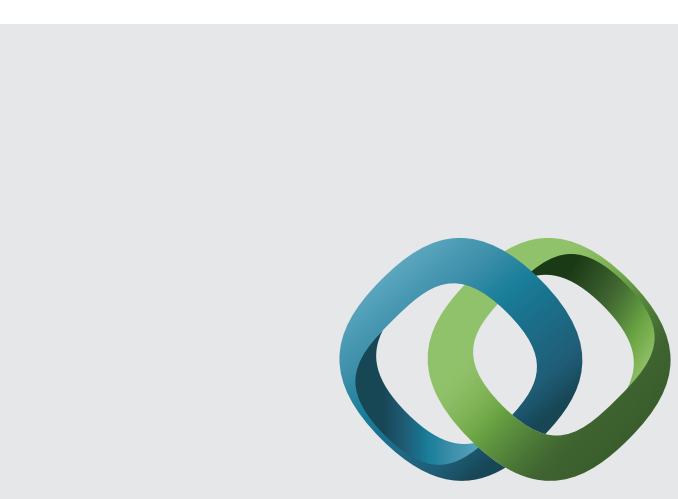

\section{Hindawi}

Submit your manuscripts at

http://www.hindawi.com
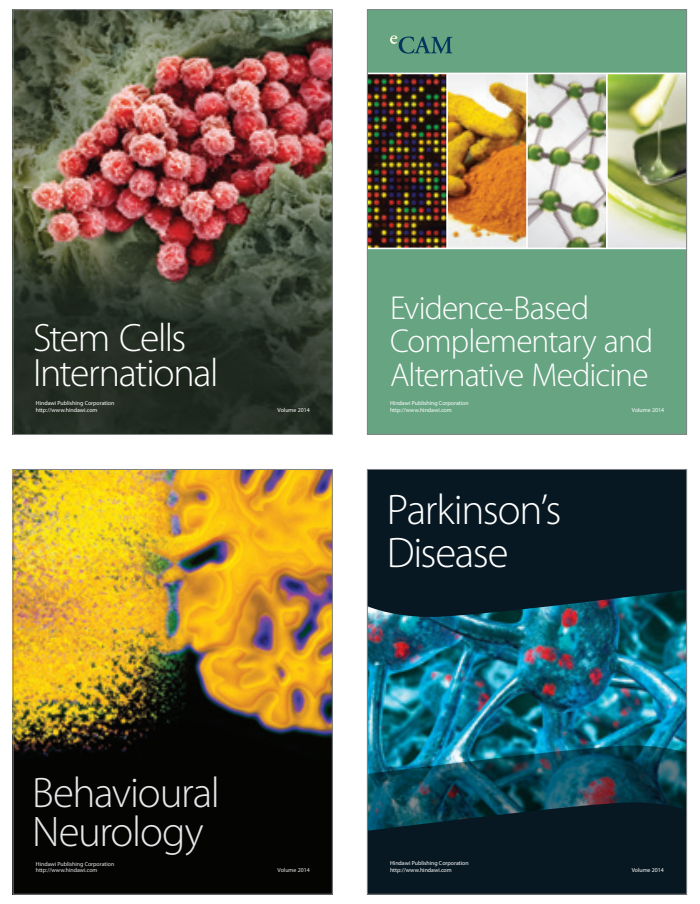
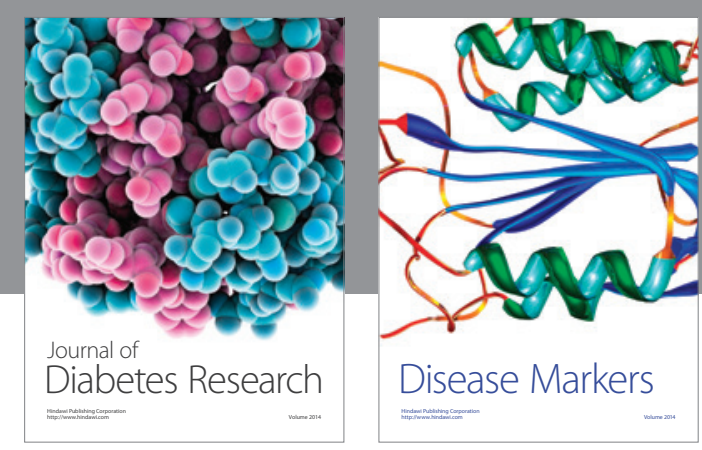

Disease Markers
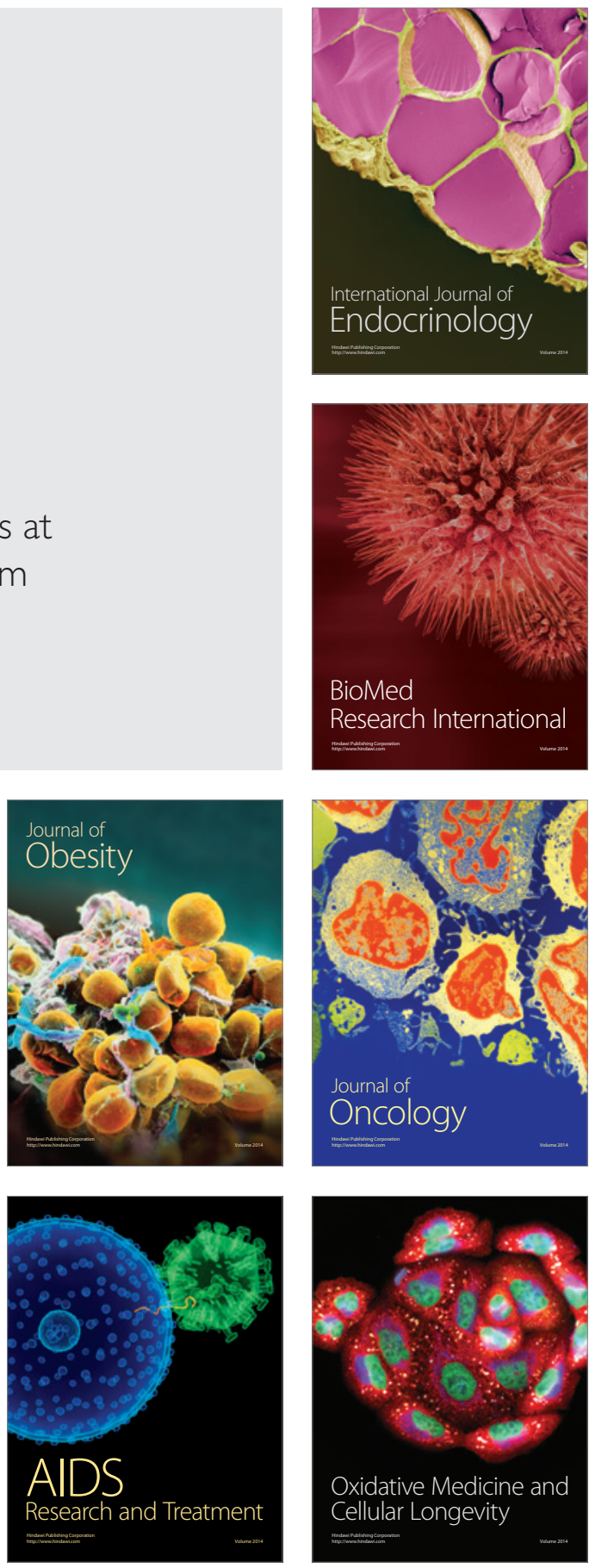